Discussion: Lower autonomy and greater risk-taking found among teenagers with same-sex partners echo the findings of research elsewhere (mainly the US). Further research on underlying psychosocial factors is required.

\section{CIGARETTE AND ALCOHOL CONSUMPTION AMONG YOUNG PEOPLE IN ENGLAND: FINDINGS FROM THE SMOKING, DRINKING AND DRUG USE SURVEY 2008}

D Jotangia, E Fuller. Health Research Group, National Centre for Social Research, London, UK

\section{doi:10.1136/jech.2009.096719n}

Background: The Smoking, Drinking and Drug Use Survey (SDD) among secondary school children in England provides national estimates for the proportions of young people (aged 11-15) who smoke, drink alcohol or take illegal drugs. The survey findings are used to monitor progress towards government targets for reducing smoking and alcohol misuse among teenagers. Until October 2007, it was illegal to sell tobacco products to children under the age of 16 in England. Evidence from SDD 2006 showed that despite this, children under the age of 16 could easily obtain cigarettes from shops and vending machines. From October 2007, it became illegal to sell cigarettes to young people under the age of 18. SDD 2008 is the first survey post implementation of this legislation which can provide empirical evidence against which to assess the impact of this legislative change upon young people's ability to buy cigarettes; impact upon smoking prevalence and assess whether young people have changed how and where they obtain cigarettes. The Chief Medical Officer launched a public consultation on young people's drinking, which advised that young people under the age of 16 should not drink alcohol because of the health risks associated with it. Evidence from previous SDD reports has shown a downward trend in the proportion of young people who have ever drunk alcohol; 61\% in 2003, 54\% in 2007. Results from the 2008 survey will monitor these trends and provide key estimates on young people's alcohol consumption and behaviour.

Methodology: A representative sample of pupils in school years 7 to 11, were drawn from all state-funded and independent secondarylevel schools across England. A random probability sample of both schools and pupils within schools was selected for participation in SDD 2008 and 7798 pupils in 253 schools participated. Pupils completed a self-completion questionnaire which included detailed questions about smoking and drinking consumption, and general questions about relevant behaviours, attitudes and knowledge.

Results: This is first report in the survey series to present nationally representative findings assessing the impact of regulating the sale of tobacco upon young people's smoking behaviour. Trend data about alcohol intake and frequency of consumption will also be presented. The SDD 2008 findings are currently under embargo prior to publication in July 2009.

Conclusions: The survey is a key data resource in the planning of young people's health services, education programmes and policy initiatives in England.

\section{Methods}

\section{A NOVEL APPROACH TO TESTING THE LIFECOURSE EFFECT OF BODY SIZES ON BLOOD PRESSURE IN LATER LIFE USING PARTIAL LEAST SQUARES REGRESSION}

\footnotetext{
${ }^{1,2}$ Y-K Tu, ${ }^{1} \mathrm{~A}$ Woolston, ${ }^{1} \mathrm{P}$ Baxter, ${ }^{1} \mathrm{MS}$ Gilthorpe. ${ }^{1}$ Biostatistics Unit, Centre for Epidemiology and Biostatistics, University of Leeds, Leeds, UK; ${ }^{2}$ Leeds Dental Institute, University of Leeds, Leeds, UK
}

doi:10.1136/jech.2009.096719o

Introduction: Recent studies claimed that postnatal catch-up growth might have a stronger impact on health in later life than birth size. As growth is a continuing process in lifecourse, the challenge is therefore to tease out the impact of body size in difference critical phases of lifecourse. One problem is that it is impossible to use ordinary least squares regression to differentiate the effects of birth sizes, growth and current body sizes, because growth is generally defined as the difference between birth and current size. The aim of this presentation is to propose a novel approach to testing lifecourse effects of body sizes on health outcomes using partial least squares (PLS).

Materials and Methods: Longitudinal data from a cohort of 960 males and 834 females recruited in Philippines during 1983-4 were used for statistical analysis. Body weight $z$-scores were used as the measure for body sizes, and the outcomes were systolic (SBP) and diastolic (DBP) blood pressure measured in 2002. PLS regression was used to test the impact of birth weight $z$-scores, change in body weight z-scores during different stages of growth, and current body weight $z$-scores on SBP and DBP.

Results: For SBP, birth size had a small negative association and the change in z-scores between age 1 and 2 had a stronger positive association than the change between birth and age 1. Growth after age 8 had a much stronger association than early growth, but current body size had the largest association. For DBP, birth size had a small positive association, and early growth had small associations in females. Growth after age 8 had a much stronger association than early growth, but current body size still had the largest positive association. In contrast, early growth had stronger associations with DBP in males, whilst current body size still had the largest association. Conclusion: PLS regression estimates the associations between birth sizes, changes in body sizes at different phases of lifecourse and current body sizes simultaneously, and therefore misinterpretation such as reversal paradox can be avoided. Results from PLS analysis suggested that current body size had the largest positive association with SBP and DBP, whilst birth size had small negative or positive associations with blood pressure. Later growth in childhood and adolescence had stronger associations with SBP than early growth before age 2 , but the associations between growth in body sizes and DBP were more complex.

\section{THE PREFERENCE EFFECT IN AN UNBLINDED HEALTH PROMOTION INTERVENTION TRIAL: HOW IMPORTANT IS IT?}

${ }^{1} \mathrm{C}$ Hayes, ${ }^{2} \mathrm{C}$ Collins, ${ }^{3} \mathrm{M}$ Geary, ${ }^{4} \mathrm{CC}$ Kelleher. ${ }^{1}$ Department of Public Health, Dr Steevens' Hospital, Dublin 8, Ireland; '2rish College of General Practitioners, Dublin, Ireland; ${ }^{3}$ Rotunda Hospital, Dublin, Ireland; ${ }^{4}$ School of Public Health and Population Science, University College Dublin, Dublin, Ireland

\section{doi:10.1136/jech.2009.096719p}

Background: Maternal smoking is one of the few potentially preventable factors associated with miscarriage, complications of pregnancy, low birth weight, perinatal death and poorer long term growth, development and health of the child. Health promotion initiatives pose a particular challenge especially for disadvantaged women. The preference effect, whereby people support an intervention because it conforms with their own understanding of a behaviour or disease under study, is one such determinant and is especially relevant to unblinded trials.

Objective: To determine whether assignment to intervention or control group and smoking status affected participation in an unblinded intervention study, one of the aims of which was to identify the key determinants of sustained effective brief intervention for smoking cessation in pregnancy and post partum.

Participants: The sample comprised a cohort of 1000 disadvantaged pregnant smokers who were followed up at five time pointstwice during pregnancy at the hospital, once directly after birth and at two subsequent public health nurse visits (3-4 and 7-9 months post partum). 